\title{
Insight into the biology of Macrophage Migration Inhibitory Factor (MIF) revealed by the cloning of its cell surface receptor
}

\author{
Lin Leng ${ }^{1}$, Richard Bucala ${ }^{1}$ \\ ${ }^{I}$ Departments of Medicine and Pathology, Yale University School of Medicine, The Anlyan Center, S525, 300 Cedar Street, New \\ Haven CT 06520-8056, USA
}

The recent cloning of MIF receptor fills an important gap in our understanding of the molecular biology and immunology of MIF. The MIF receptor, like MIF, does not fall into any established family of protein mediators, providing both new challenges and opportunities for the structural and functional analysis of MIF signal transduction.

Cell Research (2006) 16:162-168. doi:10.1038/sj.cr.7310022; published online 13 February 2006

Keywords: MIF, MIF receptor, ERK-1/2

\section{Introduction}

The protein mediator known as macrophage migration inhibitory factor (MIF) is considered to be one of the first cytokines to be discovered. In the late 1950s, "MIF" described an activity elaborated by activated lymphocytes that arrested the random movement of monocytes/macrophages [1]. This activity correlates with delayed-type hypersensitivity in vitro, and it induced significant interest among immunologists for representing a soluble, but non-immunoglobulin, factor that could be studied and manipulated in vitro. By 1966, John David and Barry Bloom independently described that MIF was most likely a protein that was secreted into the conditioned medium of activated lymphocytes [2,3], and immunologists soon measured MIF's eponymous activity by a capillary tube assay. However, MIF was difficult to isolate or clone, so as various other lymphokines (T cell growth factor/IL-2), monokines (endogenous pyrogen/IL-1; tumor necrosis factor/lymphotoxin), and interferons were systematically cloned and produced recombinantly and additional proteins, such as interferon-g and IL-4, were also observed to

Correspondence: Lin Leng

E-mail:Lin.Leng@Yale.edu

Abbreviations: Alexa-MIF (Alexa-488-modified MIF); ERK-1/2 (extracellular signal-related kinase); KO (gene knock-out); LPS (lipopolysaccharide); MAPK (mitogen-activated protein kinase); MIF (macrophage migration inhibitory factor); RIP (regulated intramembrane proteolysis); sCD74 (soluble CD74 ectodomain); TLR-4 (Toll-like receptor 4). exhibit migration inhibitory activity, immunologic interest in MIF waned. It wasn't until 1989 that a discrete gene sequence was determined to be associated with MIF [4] and in 1993, pure recombinant protein became available for biologic and structure-function studies [5]. The threedimensional crystal structure of MIF was solved by three laboratories in 1996, thus revealing a new protein fold and structural superfamily with MIF as its defining member [6-8]. These studies also provided insights into the likely native form of the protein - a homotrimer with a MW of $37.5 \mathrm{kDa}$ (Figure 1).

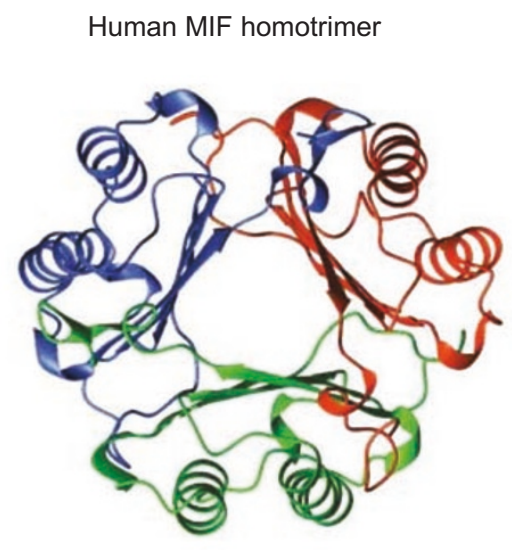

Figure 1 Three-dimensional ribbon diagram of human MIF, revealing its homotrimeric subunit structure. From ref [7]. Each color denotes one monomer. 
The crystal structure determination of MIF showed its marked topologic similarity with certain small bacterial tautomerases such as 4-oxalocrotonate tautomerase. Independent and simultaneous studies by Rorsman and colleagues, who were investigating the enzymatic pathway leading to melanin production, provided evidence that MIF catalyzed the tautomerization of the non-physiologic stereoisomer $D$-dopachrome, a precursor of melanin [9]. Although no physiologic enzymatic activity could be identified, this observation fueled interest into the possible catalytic function of MIF, which together with the lack of molecular information about a cell surface receptor, suggested that MIF mediated its immunologic action via an enzymatic reaction [10]. Site-directed mutagenesis of the protein's N-terminal, putative catalytic proline eliminated bioactivity in some studies $[11,12]$, but not in others [1315]. This cast further doubt on the requirement for a cell surface receptor.

The powerful neutralizing action of soluble anti-MIF antibody, both in vitro and in vivo, together with the retention of MIF biologic activity in many studies of genetically-engineered mutant proteins lacking catalytic activity prompted us to continue the pursuit of a specific, cell surface receptor for MIF. We first addressed this question by studying the signaling pathways that might be activated by MIF stimulation of cells. Beforehand, MIF's effect on cells had been defined biologically; the mediator had significant pro-inflammatory and activating properties on immune cells such as the augmentation of cytokine and NO production and enhanced killing of intracellular pathogens. Many of these actions were attributed to a broad, counter-regulating action on glucocorticoid-mediated immunosuppression of inflammatory responses [16].

We examined phosphorylated substrates within the cytosolic lysates of MIF-stimulated cells. To obviate concerns about contaminating LPS- which has the property of broadly activating diverse signaling pathways in monocytes/macrophages, we performed initial work in MIFstimulated fibroblasts, which are cells that are relatively insensitive to LPS. We detected an MIF-induced increase in the phosphorylation and activation of the p44/p42 extracellular-signal-regulated kinases (ERK-1/2) family of MAP kinase proteins but not the p38 and SAPK family members [17]. Small molecule inhibitors of ERK-1/2 also were observed to inhibit MIF-dependent ERK-1/2 phosphorylation. The ERK-1/2 proteins are proline-directed, serine/threonine kinases that are generally activated in response to growth or differentiation signals $[18,19]$. ERK phosphorylation was detected as early as $30 \mathrm{~min}$ after MIF addition but, remarkably, was sustained for a period of at least $24 \mathrm{~h}$. This was surprising because in physiological processes mediated by Ras protein, ERK-1/2 activation is almost always terminated in $<90 \min [19,20]$. Notable exceptions include transformation by oncogenic ras, and co-stimulation pathways mediated by prolonged integrin ligation [21-23].

An important concept regarding ERK-1/2 signal transduction is that the temporal pattern of ERK-1/2 activation (transient versus sustained) critically determines the fate of the ensuing cellular response [19]. While ERK-1/2 kinase has been best characterized for its role in growth control, ERK-1/2 also activate several downstream effector proteins that are involved in the inflammatory response, such as transcription factors ( $c-m y c, \mathrm{NF}-\mathrm{kB}$, and Ets), cytoskeletal proteins mediating membrane activation and phagocytosis, and other protein kinases [19, 24]. Activation of cells by MIF leads to the phosphorylation of ELK-1 [17], which is a member of the Ets family of transcription factors that has gained recent interest because of its role in mediating the expression of Toll-like receptor 4 (TLR-4) [25]. TLR-4 is the receptor for endotoxin (LPS), and it is expressed in reduced levels in mice genetically-deficient in MIF [25]. Among ERK-1/2 effector proteins, cytoplasmic phospholipase $\mathrm{A}_{2}\left(\mathrm{cPLA}_{2}\right)$ is an important component of the inflammatory cascade and its enzymatic product, arachidonic acid, is the precursor for the synthesis of prostaglandins and leukotrienes [26]. Arachidonic acid also activates the $c$-jun $\mathrm{N}$-terminal kinase, which is required for the efficient translation of TNF $\alpha$ mRNA [27]. cPLA 2 is an important regulatory target for the anti-inflammatory action of glucocorticoids [28], and our experiments showed that MIF could fully "override" glucocorticoid inhibition of cPLA activation [17], thus providing one node of molecular control by which MIF-via sustained ERK-1/2 activation - counter-regulates glucocorticoid effects on macrophages. Another target of interaction between MIF and glucocorticoids has been provided by recent studies of MAPK phosphatase (MPK-1) which acts to de-phosphorylate ERK-1/2 and down-regulate the MAPK activation response [29]. MIF downregulates MPK-1, which may contribute to the sustained pattern of MIF activation and glucocorticoid insensitivity of cells.

\section{Cloning of the MIF receptor}

The specific ability of MIF to induce cellular ERK-1/2 activation [17] strongly affirmed to us that MIF interacts with a specific cell surface receptor. Our initial attempts to quantify high-affinity cell surface binding sites for MIF were frustrated by the loss of MIF biologic activity upon radioiodination with either tyrosine-directed (lactoperoxidase) or amine-directed (Bolton-Hunter) methodologies. Radioiodination likely results in the oxidation of MIF's cysteine residues, which may need to be in a reduced state 
for cytokine activity [30]. MIF that was metabolically labeled with ${ }^{35} \mathrm{~S}$ and purified biochemically could be shown to bind to monocytes in a saturable and competitive manner, but the specific activity of binding was too low for the further purification or selection of high-affinity binding sites. We succeeded in creating a fluorescently-labeled MIF species that showed retention of biologic activity (i.e. proliferation, inhibition of apoptosis) by linking MIF to the dye Alexa-488 (8-(6-aminohexyl)amninoademosine3',5'cyclicmonophosphate, bis(triethylammonium)) using mild reaction conditions optimized to produce an average ligand /MIF (homotrimer) ratio of 1:1.

We observed the specific binding of Alexa-MIF to a subpopulation of human THP-1 monocytes by flow cytometry (Figure 2) [31]. High affinity binding sites were increased by the pre-activation of THP-1 monocytes with interferon- $\gamma$. Quantitative binding studies performed with increasing concentrations of MIF and analyzed by flow cytometry provided data consistent with two apparent classes of cell surface receptors on THP-1 monocytes. The higher affinity binding activity showed a $\mathrm{Kd}$ of $3.7 \times 10^{-8} \mathrm{M}$ , and the lower affinity binding showed a $\mathrm{Kd}$ of $3.5 \times 10^{-7}$ M. Confocal microscopy and direct visualization of human THP- 1 monocytes at $4^{\circ} \mathrm{C}$ also showed surface binding of Alexa-MIF, and cell-bound Alexa-MIF was internalized
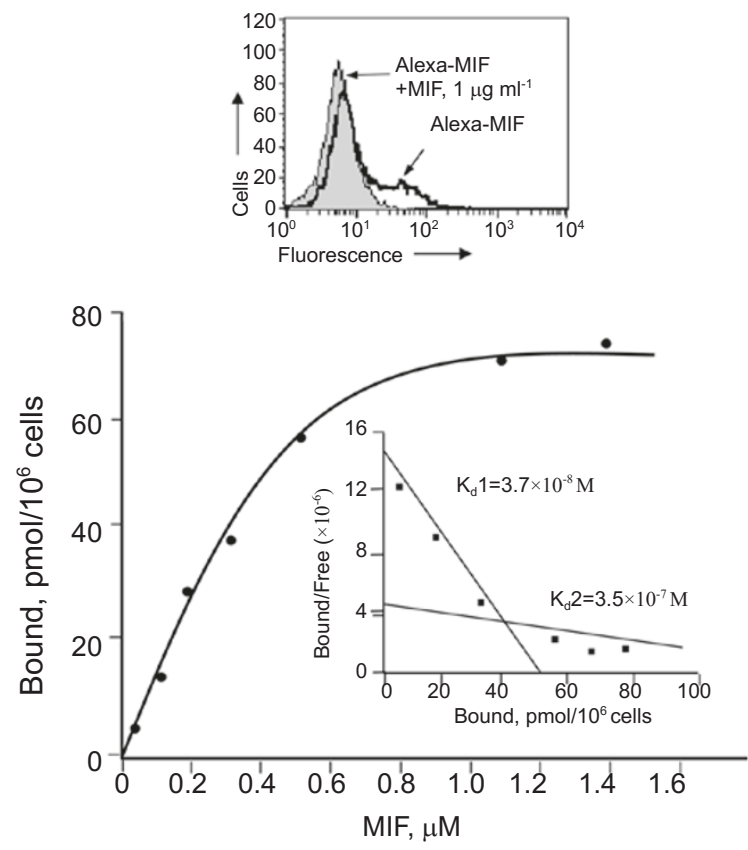

Figure 2 Co-culture of THP-1 human monocytes with IFNã produces a subpopulation of cells with high affinity binding for MIF. Binding data were generated using CellQuest Software (Becton Dickinson, San Jose, CA) from flow data of total THP-1 cells incubated with Alexa-MIF and increasing concentrations of MIF. upon shifting temperature to $37^{\circ} \mathrm{C}$.

To identify the MIF cell surface receptor, we employed an expression cloning strategy based on high-speed fluorescence-activated cell sorting and enrichment of positivelystaining clones by re-amplification (Supplementary 1). We constructed a mammalian expression library using cDNA prepared from human THP-1 monocytes. Library aliquots were transfected into COS-7 cells, and the cells which stained with Alexa-MIF purified by fluorescence cell sorting. The cDNA clones then were amplified and retransfected for additional rounds of cell sorting. After four rounds of FACS selection, single colonies were prepared in $E$. coli and the plasmid DNA isolated. We sequenced 50 clones and found that the majority encoded CD74, the cell surface form of the Major Histocompatibility Class II-associated invariant chain, that is a $31-41 \mathrm{kDa}$ Type II transmembrane protein (Figure 3). Invariant chain has been well-studied for its role as a Major Histocompatibility Class II chaperone [32], and the cell surface CD74 form has a previously described role in immune cell co-stimulation $[33,34]$. It is notable that like MIF, cell surface CD74 is expressed as a homotrimer [35].

To assess the functional significance of MIF binding to CD74, we examined the capacity of MIF to stimulate p44/p42 phosphorylation in primary macrophages obtained from mice genetically deficient in CD74 [36]. MIF increased the phosphorylation of the p44/p42 (ERK-1/2) proteins in $\mathrm{CD} 74^{+/+}$, but not in $\mathrm{CD} 74^{-/ /}$macrophages, and there was no MIF-dependent increase of $\mathrm{PGE}_{2}$ production in the $\mathrm{CD} 74^{-/}$macrophages. Transfection of a mammalian CD74 expression plasmid into the COS-7 cell line (which are $\mathrm{CD} 4^{-/}$) also reconstituted MIF induction of ERK-1/2 phosphorylation [31].

We measured the binding constant between recombinant CD74 ectodomain (sCD74 ${ }^{73-232}$ ) and MIF by surface plasmon resonance (BIAcore), which quantifies real-time binding interactions by changes in the refractive index of a biospecific surface. BIAcore analysis of the binding interaction between MIF and $\mathrm{sCD} 74^{73-232}$ revealed an equilibrium $\mathrm{Kd}$ of $9.0 \times 10^{-9}-2.3 \times 10^{-10}$, depending on which molecule of the pair was immobilized to the solid surface. This $\mathrm{Kd}$ value may be somewhat lower than the dissociation constant in vivo because native CD74 is a trimer and removal of the CD74 transmembrane domain (to facilitate cloning and expression) may inhibit native CD74 oligomerization [37]. Nevertheless, it is well within the range expected for the nanomolar concentrations of MIF that exist in the circulation.

These studies thus established that MIF binds to cells by a specific interaction with the extracellular domain of CD74 to initiate ERK-1/2 activation. This finding was unexpected because the molecular biology of CD74 had been 

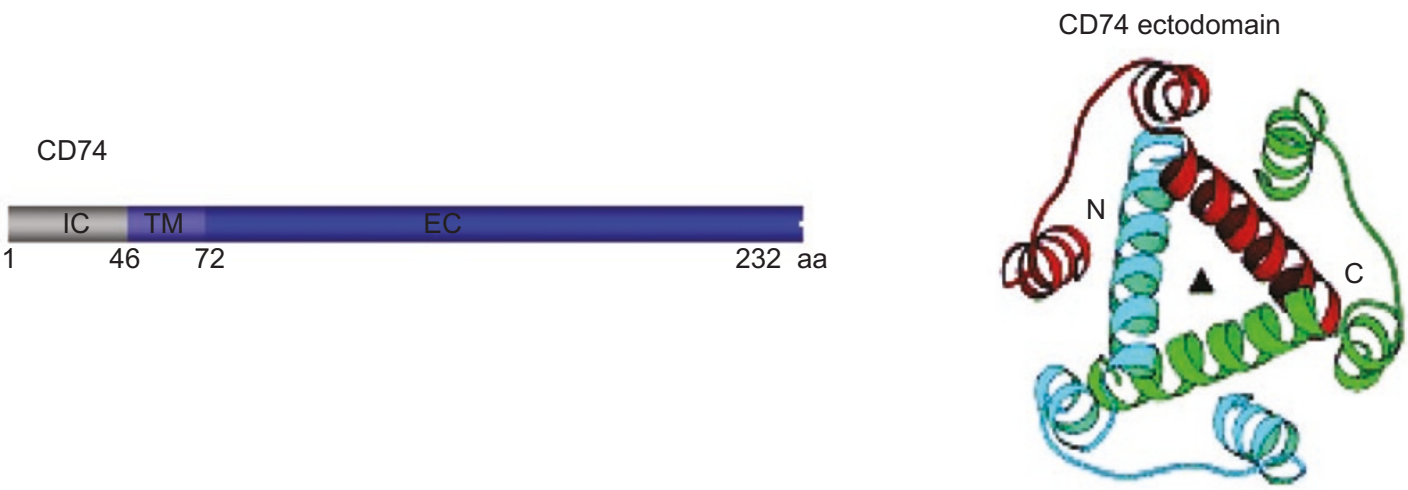

Figure 3 Diagram of human CD74 showing its intracytoplasmic (IC), transmembrane ( TM), and extracellular domains (EC). Ribbon diagram of CD74 derived from its three-dimensional crystal structure [35]. Each color denotes one monomer.

so well-defined in the context of its intracellular form as the Class II invariant chain. Nevertheless, it is known that approximately $2-5 \%$ of cellular Ii is expressed on the cell surface as CD74 [38,39] and that CD74 expression occurs independently of class II in diverse cell types including monocytes, B cells, activated T cells, and fibroblasts [40, 41]. CD74 surface expression is also known to be regulated by the length of the protein's N-terminal, intracellular domain, which varies depending on which of two in-phase initiation codons are utilized [42]. Whether this differential translation of CD74 mRNA mediates cellular sensitivity to MIF will be important to investigate.

\section{How does the MIF receptor (CD74) explain MIF action?}

An evident question is the extent to which CD74-KO mice mimic the phenotype of MIF deficiency. CD74-KO mice were created almost ten years ago [36], and MIF-KO mice have been developed recently by different targeting strategies [43-46]. The MIF KO's display strain-dependent variation in immunologic phenotype, but the recent description of an impaired $\mathrm{T}_{\mathrm{H}} 2$ response in these mice mimics what has been reported in mice deficient in CD74 [47, 48]. CD74-KO mice have developmental abnormalities in their B cell compartment, although Class II expression appears unchanged. Nevertheless, the B cell abnormalities in CD74-deficient mice extend beyond what may be expected from the protein's function as a class II chaperone [36]. Idit Shachar and her colleagues have followed up on these observations by studying more the relevant role of CD74 in B cell maturation. Interestingly, the protein has been discovered to undergo regulated intramembrane proteolysis (RIP) [49, 50], which is a recently characterized pathway for the mobilization of dormant transcription factors. Transcription factors of this class are synthesized initially in an inactive form that "nest" as integral membrane precursor proteins. Following cleavage, an active domain is released from the membrane and translocates into the nucleus to activate gene transcription. RIP is known for its conservation in eukaryotic and prokaryotic systems, and it functions to control of diverse biological processes and in response to a variety of signals $[51,52]$. In most examples of RIP that have been studied, cleavage occurs by a two-step sequential proteolytic process, with the first step involving the cleavage of the extracytoplasmic segment to shorten the ectodomain to less than 30 aa. A second proteolytic event then occurs within the transmembrane domain, most likely to permit accessibility to the second protease, which releases the product from the lipid bilayer into the cytosol. To determine whether CD74 is processed by RIP, Shachar and colleagues have followed the processing and cleavage of the CD74 extracellular domain, and the release of CD74 intracellular domain followed by its translocation to the nucleus. Entry of the CD74 intracytoplasmic domain into the nucleus leads to the activation of transcription mediated by the NF-kB p65/RelA homodimer and its coactivator, $\mathrm{TAF}_{\text {II }} 105[49,53]$. To date, this CD74-dependent activation pathway has been studied exclusively in B cells, and whether there is a role for a ligand, such as MIF, in this process remains to be studied.

\section{How does MIF mediate signal transduction through its receptor?}

From a structural perspective, the signaling properties of a CD74 homotrimer are not apparent. The cytosolic domain of CD74 is only 29-46 aa in length - depending on which of two alternative, in-phase initiation codons are translated 


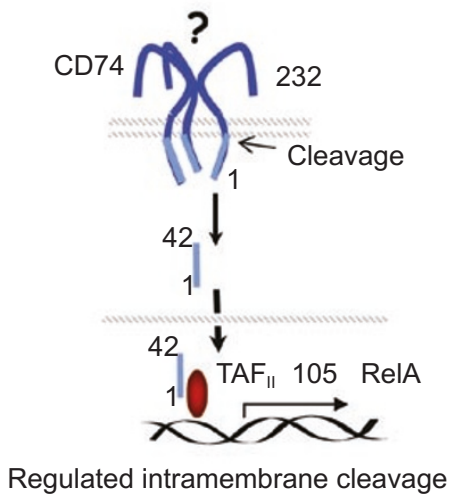

Regulated intramembrane cleavage

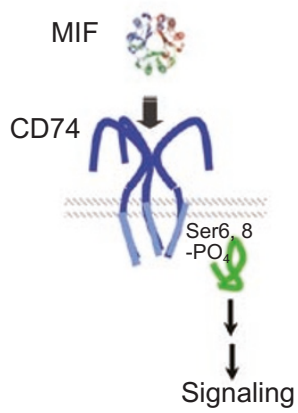

Recruitment of cytosolic proteins

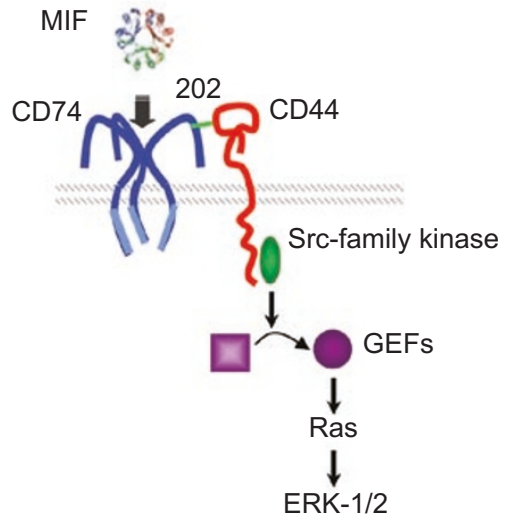

Horizontal recruitment of a membrane signaling protein

Figure 4 Proposed pathways for MIF signaling based on the known molecular biology of the MIF receptor (CD74).

[42], and it lacks homology with the catalytic domains of tyrosine or serine/threonine kinases, or with domains that might interact with non-receptor tyrosine kinases, or nucleotide binding proteins. The intracytoplasmic portion of CD74 is known to undergo phosphorylation (Ser6, Ser8), in a protein-kinase dependent manner, but evidence supporting ligand activation in this process has not been reported [54]. It nevertheless is noteworthy that recent studies have identified an accessory role for CD74 in T cell stimulation, and by a function that requires a chondroitin-sulphatedependent interaction between CD74 and CD44 [33, 34]. CD44 is a widely-expressed, polymorphic transmembrane protein with known tyrosine kinase activation properties [55]. Recent evidence in support of a molecular complex between MIF, CD74 and CD44 was provided recently by Meyer-Siegler et al. [56], who utilized an immunoprecipitation approach in cultured carcinoma cells.

Figure 4 summarizes our current hypotheses regarding the potential mechanisms for MIF signal transduction via CD74 based on the known molecular biology of these proteins. CD74 RIP is an interesting and well-worked out mechanism for signaling in B lymphocytes. Activation of the RelA/NF-kB family of transcription factors has yet been reported to be a feature of MIF action however, and MIF action in B lymphocytes has not yet been studied. The phosphorylation of the CD74 intracytoplasmic domain is strongly suggestive of a signal transduction function requiring contact with second messengers. We believe that the recruitment of a second transmembrane protein, such as CD44, is a likely mechanism for MIF signaling, especially since CD44 is known to activate Src-family kinases leading to downstream ERK phosphorylation. Receptor activation by such "horizontal" recruitment is typical of proteins that span the membrane only once, and it is the structural basis for signal transduction by several known cytokine receptors [57]. The IL-6 receptor family, for instance, does not contain a functional cytoplasmic or intracellular domain, and is incapable of transducing a signal directly. Rather, the IL-6/IL-6 receptor complex interacts with a second membrane protein, gp130, which then transduces its signal [58]. The highly polymorphic nature of the CD44 ectodomain, which additionally has a role in cell adhesion, also lends itself to cell-specific pleiotropism [59]. Such a pathway is within the expectation for MIF's broad regulatory role in cell survival and apoptosis $[46,60,61]$ and suggests that MIF's actions, which have long been considered to be extremely broad for a classical "pro-inflammatory" cytokine, may be productively regulated by the interaction between a cell surface binding protein (i.e. CD74) with a polymorphic, signal transduction molecule (CD44).

\section{Acknowledgements}

We are grateful to Dr Idit Shachar (Weizmann Instiute, Rehovot, Israel) for helpful discussions. Studies in the authors' laboratory are supported by the NIH grants AI42310, AR49610, and AR050498.

\section{References}

1 George M, Vaughn JH. In vitro cell migration as a model for delayed hypersensitivity. Proc Soc Exptl Biol Med 1962; 111:51421. 
2 David J. Delayed hypersensitivity in vitro: Its mediation by cellfree substances formed by lymphoid cell-antigen interaction. Proc Natl Acad Sci U S A 1966; 56:72-7.

3 Bloom BR, Bennett B. Mechanism of a reaction in vitro associated with delayed-type hypersensitivity. Science 1966; 153:802.

4 Weiser WY, Temple PA, Witek-Giannotti JS, Remold HG, Clark SC, David JR. Molecular cloning of a cDNA encoding a human macrophage migration inhibitory factor. Proc Natl Acad Sci U S A 1989; 86:7522-6.

5 Bernhagen J, Calandra T, Mitchell RA, et al. MIF is a pituitaryderived cytokine that potentiates lethal endotoxaemia. Nature 1993; 365:756-9.

6 Sugimoto H, Suzuki M, Nakagawa A, Tanaka I, Nishihira J. Crystal structure of macrophage migration inhibitory factor from human lymphocyte at 2.1 A resolution. FEBS Letters 1996; 389:145-8.

7 Sun HW, Bernhagen J, Bucala R, Lolis E. Crystal structure at 2.6-A resolution of human macrophage migration inhibitory factor. Proc Natl Acad Sci U S A 1996; 93:5191-6.

8 Kato Y, Muto T, Tomura T, et al. The crystal structure of human glycosylation-inhibiting factor is a trimeric barrel with three 6-stranded beta-sheets. Proc Natl Acad Sci U S A 1996; 93:300710.

9 Rosengren E, Bucala R, Aman P, et al. The immunoregulatory mediator macrophage migration inhibitory factor (MIF) catalyzes a tautomerization reaction. Molecular Medicine 1996; 2:1439.

10 Swope MD, Lolis E. Macrophage Migration Inhibitory Factor: Cytokine, Hormone, or Enzyme. Rev Physiol Biochem Pharmacol 1999; 139:1-32.

11 Swope M, Sun HW, Blake PR, Lolis E. Direct link between cytokine activity and a catalytic site for macrophage migration inhibitory factor. EMBO J 1998; 17:3534-41.

12 Onodera S, Kaneda K, Mizue Y, Koyama Y, Fujinaga M, Nishihira J. Macrophage migration inhibitory factor up-regulates expression of matrix metalloproteinases in synovial fibroblasts of rheumatoid arthritis. J Biol Chem 2000; 275:444-50.

13 Bendrat K, Al-Abed Y, Callaway DJ, et al. Biochemical and mutational investigations of the enzymatic activity of macrophage migration inhibitory factor. Biochemistry 1997; 36:15356-62.

14 Hermanowski-Vosatka A, Mundt SS, Ayala JM, et al. Enzymatically inactive macrophage migration inhibitory factor inhibits monocyte chemotaxis and random migration. Biochemistry 1999; 38:12841-949.

15 Senter PD, Al-Abed Y, Metz CN, et al. Inhibition of macrophage migration inhibitory factor (MIF) tautomerase and biological activities by acetaminophen metabolites. Proc Natl Acad Sci U S A 2002; 99:144-9.

16 Bucala R. MIF rediscovered: cytokine, pituitary hormone, and glucocorticoid-induced regulator of the immune response. FASEB J 1996; 10:1607-13.

17 Mitchell RA, Metz CN, Peng T, Bucala R. Sustained mitogenactivated protein kinase (MAPK) and cytoplasmic phospholipase A2 activation by macrophage migration inhibitory factor (MIF). Regulatory role in cell proliferation and glucocorticoid action. J Biol Chem 1999; 274:18100-6.

18 Luttrell LM, Daaka Y, Lefkowitz RJ. Regulation of tyrosine kinase cascades by G-protein-coupled receptors. Curr Opin Cell
Biol 1999; 11:177-83.

19 Vojtek AB, Der CJ. Increasing complexity of the Ras signaling pathway. J Biol Chem 1998; 273:19925-8.

20 Marshall C. Specificity of receptor tyrosine kinase signaling: transient versus sustained extracellular signal regulated kinase activation. Cell 1995; 80:179-85.

21 Howe AK, Juliano RL. Distinct mechanisms mediate the initial and sustained phases of integrin-mediated activation of the Raf/ $\mathrm{MEK} /$ mitogen-activated protein kinase cascade. J Biol Chem 1998; 273:27268-74

22 Eliceiri BP, Klemke R, Stromblad S, Cheresh DA. Integrin alphavbeta3 requirement for sustained mitogen activated protein kinase activity during angiogenesis. J Cell Biol 1998; 140:125563.

23 Roovers K, Davey G, Zhu X, Bottazzi ME, Assoian RK. Alpha5beta1 integrin controls cyclin D1 expression by sustaining mitogen-activated protein kinase activity in growth factor-treated cells. Mol Cell Biol 1999; 10:3197-204.

24 Robinson MJ, Cobb MH. Mitogen-activated protein kinase pathways. Curr Opin Cell Biol 1997; 9:180-6.

25 Roger T, David J, Glauser MP, Calandra T. MIF regulates innate immune responses through modulation of Toll-like receptor 4. Nature 2001; 414:920-4.

26 Murakami M, Nakatani Y, Atsumi G, Inoue K, Kudo I. Regulatory functions of phospholipase A2. Crit Rev Immunol 1997; 17:225-83.

27 Swantek J L, Cobb MH, Geppert TD. Jun N-terminal kinase/stress activated protein kinase (JNK/SAPK) is required for lipopolysaccharide stimulation of tumor necrosis factor alpha (TNF-a) translation: glucocorticoids inhibit TNF-a translation by blocking JNK/SAPK. Mol Cell Biol 1997; 17:6274-82.

28 Goppelt-Struebe M, Rehfeldt W. Glucocorticoids inhibit TNF alpha-induced cytosolic phospholipase A2 activity. Biochim Biophys Acta 1992; 1127:163-7.

29 Roger T, Chanson AL, Knaup-Reymond M, Calandra T. Macrophage migration inhibitory factor promotes innate immune responses by suppressing glucocorticoid-induced expression of mitogen-activated protein kinase phosphatase-1. Eur J Immunol 2005; 35:3405-13.

30 Kleemann R, Kapurniotu A, Mischke R, Held J, Bernhagen J. Characterization of catalytic center mutants of macrophage migration inhibitory factor (MIF) and comparison to Cys81Ser MIF. Eur J Biochem 1999; 261:753-66.

31 Leng L, Metz C, Fang Y, et al. MIF signal transduction initiated by binding to CD74. J Exp Med 2003; 197:1467-76.

32 Cresswell P. Assembly, transport, and function of MHC class II molecules. Annu Rev Immunol 1994; 12:259-93.

33 Naujokas MF, Morin M, Anderson MS, Peterson M, Miller J. The chondroitin sulfate form of invariant chain can enhance stimulation of $\mathrm{T}$ cell responses through interaction with CD44. Cell 1993; 74:257-68.

34 Naujokas MF, Arneson LS, Fineschi B, et al. Potent effects of low levels of MHC class II associated invariant chain on CD4+ T cell development. Immunity 1995; 3:359-72.

35 Jasanoff A, Wagner G, Wiley DC. Structure of a trimeric domain of the MHC class II-associated chaperonin and targeting protein Ii. EMBO J 1998; 17:6812-8.

36 Shachar I, Flavell RA. Requirement for invariant chain in B cell maturation and function. Science 1996; 274:106-8. 
37 Ashman JB, Miller J. A role for the transmembrane domain in the trimerization of the MHC Class II-associated invariant chain. J Immunol 1999; 163:2704-12.

38 Sant AJ, Cullen SE, Schwartz BD. Biosynthetic relationships of the chondroitin sulfate proteoglycan with Ia and invariant chain glycoproteins. J Immunol 1985; 135:416-22.

39 Sant AJ, Cullen SE, Giacoletto KS, Schwartz BD. Invariant chain is the core protein of the Ia-associated chondroitin sulfate proteoglycan. J Exp Med 1985; 162:1916-34.

40 Koch H, Harris AW. Differential expression of the invariant chain in mouse tumor cells: relationship to B lymphoid development. J Immunol 1984; 132:12-5.

41 Henne C, Schwenk F, Koch N, Möller P. Surface expression of the invariant chain (CD74) is independent of concomitant expression of major histocompatibility complex class II antigens. Immunol 1995; 84:177-82.

42 Arunachalam B, Lamb C, Cresswell P. Transport properties of free and MHC class II-associated oligomers containing different isoforms of human invariant chain. Int Immunol 1993; 6:451.

43 Bozza M, Satoskar AR, Lin G, et al. Targeted disruption of migration inhibitory factor gene reveals its critical role in sepsis. J Exp Med 1999; 189:341-6.

44 Honma N, Kosecki H, Akasaka T, et al. Deficiency of the MIF gene has no significant effect on endotoxaemia. Immunology 2000; 100:84-90.

45 Fingerle-Rowson G, Satoskar A, Bucala R. Macrophage migration inhibitory factor (MIF)-deficient mice. In: Cytokine Knockouts (G. Fantuzzi, Ed.). Humana Press: Totowa NJ 2003.

46 Fingerle-Rowson G, Petrenko O, Netz CN, et al. The p53-dependent effects of macrophage migration inhibitory factor revealed by gene targeting. Proc Natl Acad Sci U S A 2003; 100:9354-9.

47 Mizue Y, Ghani S, Leng L, et al. Role for Macrophage Migration Inhibitory Factor (MIF) in Asthma. Proc Natl Acad Sci U S A 2005; 102:14410-5.

48 Topilski I, Harmelin A, Flavell RA, Levo Y, Shachar I. Preferential Th1 immune response in invariant chain-deficient mice. J Immunol 2002; 168:1610-7.

49 Matza D, Kerem A, Medvedovsky H, Lantner F, Shachar I. Invariant chain-induced $\mathrm{B}$ cell differentiation requires intramembrane proteolytic release of the cytosolic domain. Immunity 2002; 17:549-60.

50 Matza D, Kerem A, Shachar I. Invariant chain, a chain of command. Trends in Immunology 2003; 24:264-8.

51 Brown MS, Ye J, Rawson RB, Goldstein JL. Regulated intramembrane proteolysis: A control mechanism conserved from bacteria to humans. Cell 2000; 100:391-8.

52 Urban S, Freeman M. Intramembrane proteolysis controls diverse signalling pathways throughout evolution. Curr Opin Gen Dev 2002; 12:512-8.

53 Matza D, Wolstein O, Dikstein R, Shachar I. Invariant chain induces $\mathrm{B}$ cell maturation by activating a $\mathrm{TAF}_{\mathrm{II}} 105-\mathrm{NF}-\mathrm{kB}-\mathrm{de}-$ pendent transcription program. J Biol Chem 2001; 276:272036.

54 Anderson HA, Bergstralh DT, Kawamura T, Blauvelt A, Roche PA. Phosphorylation of the invariant chain by protein kinase $\mathrm{C}$ regulates MHC class II trafficking to antigen-processing compartments. J Immunol 1999; 163:5435-43.

55 Lesley J, Hyman R, Kincade P. CD44 and its interaction with extracellular matrix. Adv Immunol 1993; 54:271-335.

56 Meyer-Siegler KL, Leifheit EC, Vera PL. Inhibition of macrophage migration inhibitory factor decreases proliferation and cytokine expression in bladder cancer cells. BMC Cancer 2004; 4.

57 Stroud RM, LaPorte S, Wells JA. Cytokine-receptor signaling at the structural level. In: Cytokine Reference Vol 1: Ligands (J. J. Oppenheim and M. Feldmann, Eds.). Academic Press: San Diego, 2001.

58 Taga T, Kishimoto T. Gp130 and the interleukin-6 family of cytokines. Annu Rev Immunol 1997; 15:797-819.

59 Turley EA, Noble PW, Bourguignon LYW. Signaling properties of hyaluronan receptors. J Biol Chem 2002; 277:4589-92.

60 Hudson JD, Shoaibi MA, Maestro R, et al. A proinflammatory cytokine inhibits p53 tumor suppressor activity. J Exp Med 1999; 190:1375-82.

61 Mitchell RA, Liao H, Chesney J, et al. Macrophage migration inhibitory factor (MIF) sustains macrophage proinflammatory function by inhibiting $\mathrm{p} 53$ : regulatory role in the innate immune response. Proc Natl Acad Sci U S A 2002; 99:345-50. 